\title{
Polariton Spectrum Subject to Bose-Einstein Condensate of Excitons
}

\author{
Yu. D. Zavorotnev', O. Yu. Popova ${ }^{2}$ \\ ${ }^{1}$ Donetsk Institute for Physics and Engineering named after A. A. Galkin, R. Luxemburg Str., Donetsk, Ukraine \\ ${ }^{2}$ Donetsk State Technical University, Shybankova Square, Krasnoarmiysk, Ukraine
}

\section{Email address:}

zavorotnev.yurii@mail.ru (Yu. D. Zavorotnev),ooo_lga@mail.ru (O. Yu. Popova)

\section{To cite this article:}

Yu. D. Zavorotnev, O. Yu. Popova. Polariton Spectrum Subject to Bose-Einstein Condensate of Excitons. Journal of Photonic Materials and Technology. Vol. 1, No. 1, 2015, pp. 10-14. doi: 10.11648/j.jmpt.20150101.12

\begin{abstract}
The diagonalization of the two-body Hamiltonian has been performed by the Green function method and the polariton spectrum has been found. The result is analogous to that found by the Bogolyubov-Tyablikov method of diagonalization. Besides, the spectrum of Bose-Einstein condensate (BEC) of the excitons has been calculated. It is shown how the spectrum of polaritons is re-normalized when the BEC excitons are present.
\end{abstract}

Keywords: Polariton, Exciton, Bose-Einstein Condensate, Green Function, Spectrum

\section{Introduction}

The appearance of powerful sources of coherent radiation provides formation of the exciton density of about $10^{17}-10^{18}$ $\mathrm{cm}^{-3}$ in the crystals. At the same time, the collective properties of excitons become substantial. As excitonic excitations of a crystal can be considered as bosons with a reasonable degree of accuracy, Bose-Einstein condensation should be registered at low temperature and repulsive character of exciton-exciton interaction, when the kinetic momentum $k$ corresponds to the minimum of the exciton branch [1-7].

Despite numerous papers [8-11] reporting that the exciton condensation has been realized at last, the final consensus has not been reached yet. That is why the search for new indirect evidences of the existence of BEC excitons stays of immediate interest. It should be noted that formation of BEC would affect all the characteristics of the crystal, especially the polariton spectrum.

Polariton approach is widely used when studying nonlinear effects in both semiconductors and molecular crystals [12]. It is usually supposed that the quadratic terms of Hamiltonian related to photon-exciton interaction are large. So, diagonalization of the terms is carried out by Bogolyubov-Tyablikov transform resulting in the polariton spectrum. The rest of the terms of the third order and higher describe varied non-linear effects, in particular, Raman scattering (RS) etc. The approach suggests that all the Hamiltonian terms of the order higher of the second one are small to be accounted by the perturbation theory. It should be noted that the technique works well at the temperatures near zero and a few or absent excitations in the initial state.

The situation can be changed when the initial state is characterized by Bose-Einstein condensate of excitons where a macroscopic number of the particles is in the same definite state. Due to this fact, single terms of the order higher than the second one can be anomalously large, with the related renormalization of the spectrum.

Besides, there are compounds where the Hamiltonian terms of the third order are larger of the quadratic ones. In this case, Bogolyubov-Tyablikov transform cannot be applied.

\section{Green Function and the Spectrum of the Condensed Excitons}

Suppose that in the crystal, on the branch $\sigma$, there are interacting condensate excitons characterized by quasimomentum $\vec{k}=0$. The Hamiltonian of the system can be written as

$$
\begin{aligned}
& H=H_{0}+V_{1} \\
& H_{0}=\sum_{\vec{k}, \mu} E_{\mu}(\vec{k}) B_{\mu}^{+}(\vec{k}) B_{\mu}(\vec{k}) \\
& V_{1}=\frac{Q}{V} B_{\sigma}^{+}(0) B_{\sigma}^{+}(0) B_{\sigma}(0) B_{\sigma}(0)
\end{aligned}
$$

where $Q$ can be substituted with the constant of the excitonexciton interaction found in [12], $E_{\mu}(\vec{k})$ is the exciton 
spectrum, $\vec{k}$ is the quasi-momentum, $\mu$ is the number of the exciton band, $B^{+}, B$ are operators of nucleation and destruction of excitons, $V$ is the volume of the crystal.

Partial Green function $N_{0}$ can be written as

$$
D_{\sigma}^{\left(N_{0}\right)}(t)=-i\left\langle T\left(\tilde{B}_{\sigma}^{N_{0}}(0, t) \tilde{B}_{\sigma}^{+N_{0}}(0,0)\right)\right\rangle_{H_{0}+V_{1}}
$$

where $\tilde{B}_{\sigma}(0, t)=\exp \left(\frac{i\left(H_{0}+V_{1}\right) t}{\hbar}\right) B_{\sigma}(0) \exp \left(-\frac{i\left(H_{0}+V_{1}\right) t}{\hbar}\right)$ are operators in Heisenberg representation; function (2) is averaged over the wavefunctions of the Hamiltonian $H_{0}+V_{1}$.

The spectrum of system (1) can be calculated by the method of the motion equations. It is known [13], that the operators in the Heisenberg representation obey the motion equation

$$
i \hbar \frac{\partial \tilde{B}_{\sigma}(0, t)}{\partial t}=\left[\tilde{B}_{\sigma}(0, t), H_{0}+V_{1}\right]
$$

Using $H_{0}$ yields

$$
i \hbar \frac{\partial \tilde{B}_{\sigma}(0, t)}{\partial t}=E_{\sigma}(0) \tilde{B}_{\sigma}(0, t)+\left[\tilde{B}_{\sigma}(0, t), V_{1}\right]
$$

Differentiation of $D_{\sigma}^{\left(N_{0}\right)}(t)$ with respect to the time results in

$$
\begin{aligned}
& {\left[i \hbar \frac{\partial}{\partial t}-N_{0} E_{\sigma}(0)\right] D_{\sigma}^{\left(N_{0}\right)}(t)=N_{0} ! \delta(t)+} \\
& +\frac{2 Q}{V} D_{\sigma}^{\left(N_{0}\right)}(t)\left[\left(N_{0}-1\right)+\left(N_{0}-2\right)+\ldots+1\right]
\end{aligned}
$$

After summation of the arithmetic progression, it can be written

$$
\left[i \hbar \frac{\partial}{\partial t}-N_{0} E_{\sigma}(0)-\frac{Q}{V} N_{0}\left(N_{0}-1\right)\right] D_{\sigma}^{\left(N_{0}\right)}(t)=N_{0} ! \delta(t)
$$

In $\omega$-representation,

$$
D_{\sigma}^{\left(N_{0}\right)}(\omega)=\frac{N_{0} !}{\hbar \omega-N_{0} E_{\sigma}(0)-\frac{Q}{V} N_{0}\left(N_{0}-1\right)}
$$

Thus, the energy of $N_{0}$ condensate excitons has the form

$$
E_{N_{0}}=N_{0} E_{\sigma}(0)+\frac{Q}{V} N_{0}\left(N_{0}-1\right)
$$

So, the chemical potential of the system $\mu$ can be easily found, being determined by

$$
\mu \approx E_{N_{0}+1}-E_{N_{0}}=E_{\sigma}(0)+2 Q n_{0}
$$

at zero temperature, where $n_{0}=N_{0} V^{-1}$ is the density of the condensate particles. The energy per a volume unit is

$$
\lim _{V \rightarrow \infty} \frac{E_{N_{0}}}{V}=n_{0} E_{\sigma}(0)+Q n_{0}^{2}
$$

It is obvious that in the case of repulsion between the excitons, $Q<0$. So, $\mu<E_{\sigma}(0)$.

It should be noted that the results analogous to (9) and (10) were reported in [14]. But the use of technique by S.T. Belyaev C.T. is hardly possible in our case, because the state of condensate characterized by the kinetic momentum $\bar{k}=0$ is not the ground state of the crystal.

\section{Spectrum of the System with Bose- Condensate of Excitons}

First we should consider, how the polariton spectrum can be calculated with using Green function. As mentioned above, the problem is solved by Bogolyubov-Tyablikov transform with account of two-body interaction only.

The quadratic Hamiltonian of the system of the crystal and the field of transverse photons is written in the form

$$
H=H_{0}+V_{1}
$$

where

$$
H_{0}=\sum_{k, j} \hbar \omega(k, j) a_{j}^{+}(k) a_{j}(k)+\sum_{k, \mu} E_{\mu}(k) B_{\mu}^{+}(k) B_{\mu}(k)
$$

is the zero Hamiltonian, $\vec{k}$ is the wavevector, $j$ is the number of photon polarization, $\mu$ is the number of the exciton branch, $a^{+}, a, B^{+}, B$ are the field operators of photons and excitons, respectively, $\hbar \omega, E$ are the spectra of photons and excitons,

$$
\begin{aligned}
& V_{1}=\sum_{k, j, \mu} T(k j \mu)\left[a_{j}(k)+a_{j}^{+}(-k)\right]\left[B_{\mu}^{+}(k)-B_{\mu}(-k)\right]+ \\
& +\frac{\omega_{0}^{2}}{4 c} \sum_{k j} \frac{1}{k}\left[a_{j}(k) a_{j}(-k)+a_{j}^{+}(k) a_{j}^{+}(-k)+2 a_{j}^{+}(k) a_{j}(k)\right]
\end{aligned}
$$

$\omega_{0}$ is the plasma frequency.

We consider Green function

$$
\begin{aligned}
& G\left(j_{1} k_{1} j_{2} k_{2} t 0\right)=-i\left\langle T\left[\tilde{a}_{j_{1}}\left(k_{1} t\right)+\tilde{a}_{j_{1}}^{+}\left(-k_{1} t\right)\right] *\right. \\
& \left.*\left[\tilde{a}_{j_{2}}\left(-k_{2} 0\right)+\tilde{a}_{j_{2}}^{+}\left(k_{2} 0\right)\right]\right\rangle_{H_{0}+V_{1}}
\end{aligned}
$$

where

$$
\tilde{a}_{j}(k)=\exp \left(\frac{i\left(H_{0}+V_{1}\right) t}{\hbar}\right) a_{j}(k) \exp \left(-\frac{i\left(H_{0}+V_{1}\right) t}{\hbar}\right)
$$

$T$ is the operator of chronological ordering, the average is performed over the ground state of Hamiltonian $\left(H_{0}+V_{1}\right)$ (10). Proceed to the representation of interaction, (13) can be rewritten as 


$$
\begin{aligned}
& G\left(j_{1} k_{1} j_{2} k_{2} t 0\right)=-i\left\langle T\left[a_{j_{1}}\left(k_{1} t\right)+a_{j_{1}}^{+}\left(-k_{1} t\right)\right] *\right. \\
& \left.*\left[a_{j_{2}}\left(-k_{2} 0\right)+a_{j_{2}}^{+}\left(k_{2} 0\right)\right] S^{V_{1}}\right\rangle_{H_{0}}^{c B}
\end{aligned}
$$

where index $c 8$ means that only bound diagrams should be accounted in (14)

$$
\begin{aligned}
& S^{V_{1}}=T \exp \left\{-\frac{i}{\hbar} \int_{-\infty}^{\infty} V_{1}(t) d t\right\} \\
& a_{j}(k t)=\exp \left(\frac{i H_{0} t}{\hbar}\right) a_{j}(k) \exp \left(-\frac{i H_{0} t}{\hbar}\right) \\
& B_{\mu}(k t)=\exp \left(\frac{i H_{0} t}{\hbar}\right) B_{\mu}(k) \exp \left(-\frac{i H_{0} t}{\hbar}\right)
\end{aligned}
$$

The operator $V_{1}(t)$ is found with substitution of $a_{j}(k)$ and $B_{\mu}(k)$ with $a_{j}(k t)$ и $B_{\mu}(k t)$ in (12). In (14), the exponent is expanded into a series to be summarized. The procedure is illustrated in Fig 1.

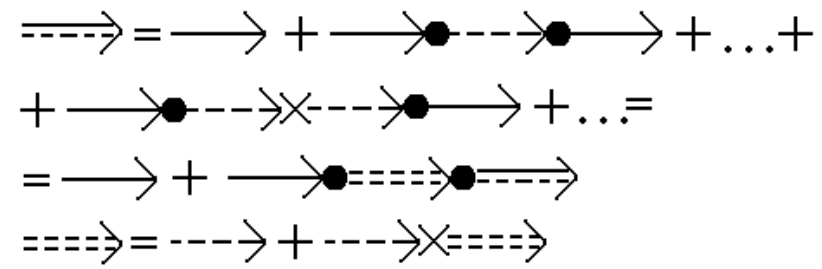

Fig. 1. Dashed and solid lines are related to zero Green functions of a photon and an exciton, respectively, the criss-cross represents the top of $\omega_{0}^{2}(2 k c)^{-1}$, the dots denote $T(k j \mu)$.

As a result, in $\omega$ representation,

$$
G(k j \omega)=D(k j \omega)+D(k j \omega) \Pi^{(1)}(k j \omega) G(k j \omega)
$$

where $D(k j \omega)$ is the zero Green function of a photon.

The solution of ( ) has the form

$$
G(k j \omega)=\frac{D(k j \omega)}{1-\Pi^{(1)}(k j \omega) D(k j \omega)}=\frac{2 \omega_{j}(k)}{\hbar\left[\varepsilon^{\perp}(k j \omega) \omega^{2}-\omega_{j}^{2}(k)\right]}
$$

$$
\varepsilon^{\perp}(k j \omega) \omega^{2}-\omega_{j}^{2}(k)=0
$$

The spectrum coincides with the spectrum of polaritons obtained in [12] by Bogolyubov-Tyablikov transform.

At $\omega \rightarrow \omega_{\rho}$, where $\rho$ is the number of the polariton branch,

$$
\begin{aligned}
& G(k j \rho \omega)=\frac{1}{\omega-\omega_{\rho}} \operatorname{Res} G(k j \omega)= \\
& =\frac{c}{v_{\phi}\left\{\varepsilon^{\perp}\left(j k \omega_{\rho}\right)+\left.\frac{\omega_{\rho}}{2} \frac{\partial \varepsilon^{\perp}(j k \omega)}{\partial \omega}\right|_{\omega=\omega_{\rho}}\right\}\left(\omega_{\rho}-\omega\right)}
\end{aligned}
$$

An analogous spectrum can be obtained, if the following Green function is calculated

$$
\begin{aligned}
& G\left(\mu_{1} k_{1} \mu_{2} k_{2} t 0\right)=-i\left\langle T\left[\tilde{B}_{\mu_{1}}\left(k_{1} t\right)+\tilde{B}_{\mu_{1}}^{+}\left(-k_{1} t\right)\right]\right. \\
& \left.\left[\tilde{B}_{\mu_{2}}\left(-k_{2} 0\right)+\tilde{B}_{\mu_{2}}^{+}\left(k_{2} 0\right)\right]\right\rangle_{H_{0}+V_{1}}
\end{aligned}
$$

The process of summation is presented in Fig. 2.

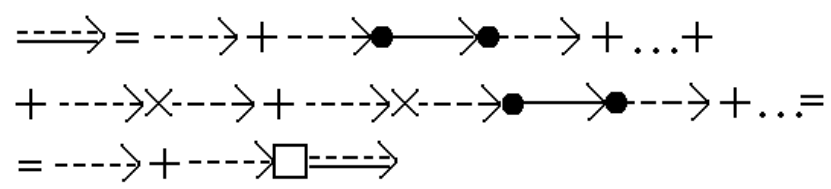

Fig. 2. Dashed and solid lines are related to zero Green functions of a photon and an exciton, respectively, the criss-cross represents the top of $\omega_{0}^{2}(2 k c)^{-1}$, the dots denote $T(k j \mu)$.

The resulting Fourier transform of the diagonal matrix element of (20) can be written in the form

$$
G(k \mu \omega)=\frac{2 E_{\mu}(k)\left[\omega^{2}(k j)+\omega_{0}^{2}-\omega^{2}\right]}{\left[E_{\mu}^{2}(k)-\omega^{2}\right]\left[\omega^{2}(k j)-\omega^{2} \varepsilon^{2}(j k \omega)\right]}
$$

The poles $G(k \mu \omega)$ coincide with the poles of $G(k j \omega)$. When $\omega$ is close to $\omega_{\rho}$, approximate $G(k \mu \omega)$ looks like

The poles (17) determine the spectrum of the system characterized by Hamiltonian $H_{0}+V_{1}(10)$. So,

$$
G(k \mu \rho \omega)=\frac{1}{\omega-\omega_{\rho}} \operatorname{Res} G(k \mu \omega)=\mid \frac{8 E_{\mu}^{2}(k) T(k j \mu)}{\left[E_{\mu}^{2}(k)-\omega_{\rho}^{2}\right]\left\{\varepsilon^{\perp}\left(j k \omega_{\rho}\right)+\left.\frac{\omega_{\rho}}{2} \frac{\partial \varepsilon^{\perp}(j k \omega)}{\partial \omega}\right|_{\omega=\omega_{\rho}}\right\}\left(\omega_{\rho}-\omega\right)}
$$

To find the spectrum of the polariton system, when BoseEinstein condensate of exciton is present in the crystal, the
Hamiltonian with the three-body terms should be diagonalized. The Hamiltonian has the form of 


$$
\begin{aligned}
& H=H_{0}+V_{1}+V_{2}+V_{3} ; \\
& H_{0}=\sum_{k, j} \hbar \omega(k, j) a_{j}^{+}(k) a_{j}(k)+\sum_{\vec{k}, \mu} E_{\mu}(\vec{k}) B_{\mu}^{+}(\vec{k}) B_{\mu}(\vec{k}) ; \\
& V_{1}=\sum_{k, j, \mu} T(k j \mu)\left[a_{j}(k)+a_{j}^{+}(-k)\right] *\left[B_{\mu}^{+}(k)-B_{\mu}(-k)\right]+\frac{\omega_{0}^{2}}{4 c} \sum_{k j} \frac{1}{k}\left[a_{j}(k) a_{j}(-k)+a_{j}^{+}(k) a_{j}^{+}(-k)+2 a_{j}^{+}(k) a_{j}(k)\right] \\
& V_{2}=\frac{Q}{V} B_{\sigma}^{+}(0) B_{\sigma}^{+}(0) B_{\sigma}(0) B_{\sigma}(0), \\
& V_{3}=\frac{1}{\sqrt{N}} \sum_{\mu j k q} R(\mu j k q)\left[a_{j}(k)+a_{j}^{+}(-k)\right] *\left[B_{\mu}^{+}(q)-B_{\mu}(-q)\right] *\left[B_{\mu}^{+}(k-q)-B_{\mu}(q-k)\right]
\end{aligned}
$$

It should be noted, that in this case, the canonical transformation, that allows a similar procedure, is absent. However, the re-normalized spectrum of the system can be found by calculation of the poles of the succeeding $\left(N_{0}+1\right)$ body Green function

$$
\begin{aligned}
& K(j k \mu 0 t 0)=-i\left\langle T\left[a_{j_{1}}(k t)+a_{j_{1}}^{+}(-k t)\right]\right. \\
& \left.B_{\sigma}^{N_{0}}(0, t) B_{\sigma}^{+N_{0}}(0,0)\left[a_{j_{2}}(-k 0)+a_{j_{2}}^{+}(k 0)\right] S^{V_{1}+V_{2}+V_{3}}\right\rangle_{H_{0}}^{c B}
\end{aligned}
$$

After the expansion of the evolution operator into a series, it is necessary to sum up the quadratic terms, to transit to the polariton variables and to perform diagonalization with respect to the three-body terms of the Hamiltonian (23). The described procedure is illustrated in Fig.3. It should be noted that two virtual processes should be accounted in the course of diagonalization. The first process includes the destruction of a polariton and the creation of a polariton and a condensate exciton (an analogue of Raman scattering). The second process implies the destruction of a polariton and a condensate exciton and the creation of a polariton.

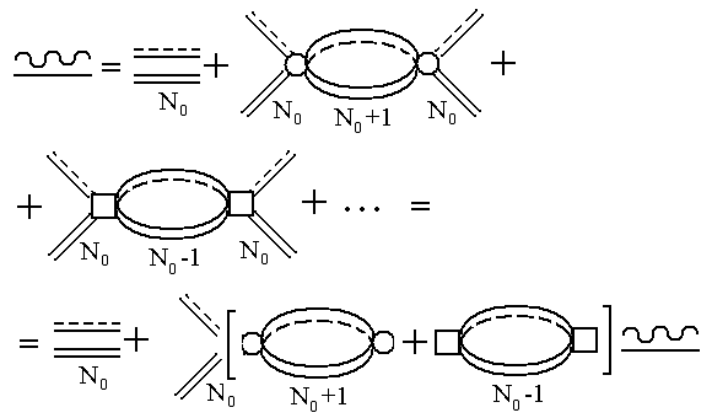

Fig. 3. To circle and square represents the tops of $R(\mu j k q)$ and $R^{*}(\mu j k q)$.

In an analytical form, the result can be written as follows

$$
\begin{aligned}
& K(j \sigma \omega)=\frac{\operatorname{Res} G(k j \omega)}{\omega_{\rho}(k)+N_{0} E_{\sigma}(0)+\frac{Q}{V} N_{0}\left(N_{0}-1\right)-\omega}+ \\
& +\frac{\operatorname{Res} G(k j \omega)}{\omega_{\rho}(k)+E_{N_{0}}(0)-\omega} * \\
& *\left[\frac{R^{2}(\sigma k k 0) \operatorname{Res} G(k \mu \omega)}{\omega_{\rho}(k)+E_{N_{0}+1}(0)-\omega}+\frac{R^{2}(\sigma k j 0) \operatorname{Res} G(k \mu \omega)}{\omega_{\rho}(k)+E_{N_{0}-1}(0)-\omega}\right] * \\
& * K(j \sigma \omega)
\end{aligned}
$$

The resulting equation allows the spectrum calculation:

$$
\begin{aligned}
& \omega^{3}-\omega^{2}\left(A_{1}+A_{2}+A_{3}\right)+\omega\left(A_{1} A_{2}+A_{1} A_{3}+A_{2} A_{3}-\right. \\
& \left.-2 R^{2}(\sigma j k 0) \operatorname{Res} G(k \mu \omega)\right)+A_{1} A_{2} A_{3}-A_{4}=0
\end{aligned}
$$

where

$$
\begin{aligned}
& A_{1}=\omega_{\rho}(k)+N_{0} E_{\sigma}+\frac{Q}{V} N_{0}\left(N_{0}-1\right) \\
& A_{2}=\omega_{\rho}(k)+\left(N_{0}+1\right) E_{\sigma}+\frac{Q}{V} N_{0}\left(N_{0}+1\right) \\
& A_{3}=\omega_{\rho}(k)+\left(N_{0}-1\right) E_{\sigma}+\frac{Q}{V}\left(N_{0}-1\right)\left(N_{0}-2\right) \\
& A_{4}=2 R^{2}(\sigma j k 0) \operatorname{Res} G(k \mu \omega) * \\
& *\left(\omega_{\rho}(k)+N_{0} E_{\sigma}+\frac{Q}{V}\left(N_{0}^{2}-N_{0}+1\right)\right)
\end{aligned}
$$

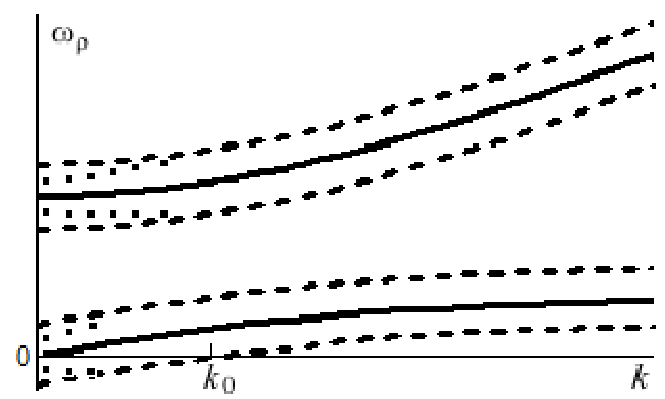

Fig. 4. Spectrum polariton in the presence of BEC excitons.

Let us suppose that the branches of $\omega_{\rho}(k)$ are far from the $\sigma$ band with the condensate excitons. So, function $\operatorname{Res} G(k \mu \omega)$ can be treated as a constant. In this case, each branch of $\omega_{\rho}(k)$ is split into three curves. One of the branches will coincide with the original one (solid lines in Fig.4), the rest of them (dashed lines in Fig.4) are located above and below by the value determined by the combination of $E_{\sigma}$ and $2 R^{2}(\sigma j k 0) \operatorname{Res} G(k \mu \omega)$. The less the difference between the magnitudes, the closer the branches. It should be noted that a part of the bottom branch from 0 to $k_{0}$ is negative and it should be omitted, being not physical.

When the Bose-Einstein condensate of excitons is located near the upper polariton branch, the magnitude of the effective three-body interaction will vary, with the maximum achieved at $k=0$ due to the presence of the resonance 
denominator in $\operatorname{Res} G(k \mu \omega)$. When the effective interaction increases, the levels approach each other, as shown by the dots in Fig.4.

\section{Conclusions}

It has been shown that the fact of formation of BEC excitons can be confirmed by the detection of the changes of the polariton spectrum.

\section{References}

[1] S.A. Moskalenko. Fiz. Tverd. Tela, vol. 4, p. 276, 1962.

[2] S.A. Moskalenko. Bose-Einstein condensation of excitons and biexcitons. - Chisinau. Shtiintsa. 1970. 166 pp.

[3] J.M. Blatt, K.W. B oer, and W. Brandt. Phys. Rev., vol. 126, p. $1691,1962$.

[4] Casella, R.C. Source. Journal of the Physics and Chemistry of Solids, vol. 24, p 19-26, 1963.

[5] L.V. Keldysh and A.N. Kozlov. Zh. Exsp. Teor. Fiz. Pis'ma, vol. 5, p. 238, 1967.
[6] L.V. Keldysh and A.N. Kozlov. Zh. Exsp. Teor. Fiz. 54, 978 (1968) [Sov. Phys. JETP 27, 521 (1968).]

[7] Chen Liang, Kong Wei, B.J. Ye, H.M. Wen, X.Y. Zhou, and R.D. Han. Fizika Nizkikh Temperatur, , vol. 37, pp. 708-714, 2011.

[8] I.H. Hakobyan, E.F. Gross, B.S. Razbirin. JETP Letters, vol. 12, p.366, 1970

[9] M.S. Brodin, D.V. Goer, M.G. Macko. JETP Letters, vol.20, p. 300, 1974.

[10] M. E. Flatte, E. Runge, H. Ehrenreich. Appl. Phys. Lett., vol. 66, pp.1313-1315, 1995

[11] A. A. High, J. R. Leonard, M. Remeika, L. V. Butov, M. Hanson, and A. C. Gossard. Nano Lett., vol. 12 (5), pp 26052609, 2012.

[12] V.M. Agranovich. The theory of excitons. M., Nauka, 1968. $328 \mathrm{p}$.

[13] A.A. Abrikosov L.P. Gorkov, I.E. Dzyaloshinskii. Methods of quantum field theory in statistical physics. Moskwa, GIFML $1962,443 \mathrm{p}$.

[14] S.T. Belyaev. JETP, vol.34, pp. 417-432, 1958. 